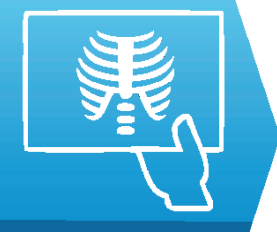

MEDICAL IMAGING

\title{
Current status of imaging diagnosis in the transplanted kidney. A review of the literature with a special focus on contrast-enhanced ultrasonography
}

1) Hemodialysis Department, $5^{\text {th }}$ Medical Clinic and Urology Department. Clinical Institute of Urology and Renal Transplantation, Iuliu Hatieganu University of Medicine and Pharmacy, ClujNapoca, Romania

2) Medical Imaging Department, "Octavian Fodor" Institute of Gastroenterology and Hepatology and Iuliu Hatieganu University of Medicine and Pharmacy, ClujNapoca, Romania

3) Urology Department, Clinical Institute of Urology and Renal Transplantation, Iuliu Hatieganu University of Medicine and Pharmacy, Cluj-Napoca, Romania

4) Medical Imaging Department, "Prof dr. Ion Chiricuta" Institute of Oncology, Cluj-Napoca, Romania

5) Morphology Department, Clinical Institute of Urology and Renal Transplantation, Cluj-Napoca. Romania

DOI: $10.15386 / \mathrm{mpr}-1536$

Manuscript received: 05.12.2019

Received in revised form: 27.01.2020

Accepted: 17.02.2020

Address for correspondence:

ramona.contis@gmail.com

This work is licensed under a Creative Commons Attribution-NonCommercialNoDerivatives 4.0 International License
Maria Ramona Bădulescu ${ }^{1}$, Mihai Adrian Socaciu², Tudor Moisoiu³, Alexandra Andries ${ }^{2,4}$, Gheorghiță Iacob ${ }^{5}$, Radu Badea ${ }^{2}$

\begin{abstract}
Objectives. Ultrasonographic scanning is currently the most widespread imaging diagnostic procedure. The method provides real-time morphological, vascular and elastographic information in a non-invasive manner. In recent years, harmonic vascular examination has become accessible using intravenous contrast agents. In urological pathology, this procedure is used in the detection and evaluation of vascular and ischemic complications, in the classification of complex cysts according to the Bosniak system, also in the renal lesions with uncertain etiology and in acute pyelonephritis for the detection of abscesses. The contrast agent (SonoVue) is angiospecific and can be used in patients transplanted immediately after surgery without adverse effects or impaired renal function. Thus, it is desirable to be used in the nephrological pathology of the renal graft and to develop diagnostic models based on the evaluation of renal microvascularization, as well as the quantitative data resulting from the graphical representation of the specific parameters. The purpose of this review is to evaluate the current state of the literature regarding the place and role of contrast substance ultrasound in the early diagnosis of acute renal graft dysfunction and to make a differential diagnosis of this pathological entity.
\end{abstract}

Method. This review quantifies the role of contrast ultrasound in the diagnosis of acute complications of the renal graft. The research was conducted based on the databases PubMed, MedScape, Cochrane, according to the search criteria such as contrast-enhanced ultrasound + kidney transplant, "time intensity curves" + "kidney transplant", filtered for the period 2004-2018.

Results. In the nephrological pathology of the renal graft, contrast-enhanced ultrasound is a valuable tool, superior to Doppler ultrasound in predicting the evolution of the renal graft, identifying very small early defects in renal microvascularization. A number of studies succeeded in identifying acute graft dysfunction, some of which establish its etiology - humoral rejection versus acute tubular necrosis. On the other hand, the contrast-enhanced ultrasound parameters do not have the ability to distinguish between cellular and humoral rejection.

Conclusions. If, at present, the histopathological examination is the only one that can differentiate with certainty the cause of acute renal graft dysfunction, we consider that contrast-enhanced ultrasound, as a non-invasive imaging technique, opens a favorable perspective for increasing the survival of the renal graft and decreasing the complications in the renal transplant. The combination of other ultrasound techniques, together with contrast-enhanced ultrasound, could lead to the development of new diagnostic models.

Keywords: kidney transplantation, imaging diagnosis, acute rejection, acute tubular necrosis, acute humoral rejection, time-intensity curve, contrast-enhanced ultrasound 


\section{Preamble}

Renal transplantation is a unique chance to a normal life for end-stage renal disease patients, because of the perfect morphological and functional replacement of the lost kidneys. It is also the only effective way of replacement of the endocrine function of the kidneys. Despite the immunosuppressive revolution of the ' $70 \mathrm{~s}$, in kidney transplant there are still events like delayed graft function (DGF) and acute rejection (AR) that are directly linked to early kidney allograft loss, if not diagnosed and treated promptly [1]. Most authors define DGF by the use of hemodialysis in the first week after the kidney transplant [2]. Acute tubular necrosis (ATN) is the most frequent cause of DGF, followed by acute humoral rejection (AHR). Because of the difference in the treatment of ATN and AHR, the correct diagnosis is essential.

Acute renal allograft dysfunction (AAD) represents the increase of serum creatinine $(\mathrm{sCr})$ level more than $1.5 \mathrm{x}$ baseline level, and/or decrease of glomerular filtration rate (GFR) more than $25 \%$, oliguria, and/or proteinuria more than $1 \mathrm{~g} /$ day [2]. There are many causes of AAD, and the differential diagnosis includes AR, urinary obstructions, bacterial and viral infections, including BK virus, vascular pathologies, calcineurin inhibitor toxicity, recurrence of primary renal disease, de novo glomerular disease, chronic allograft nephropathy. For the evaluation of kidney allograft, greyscale and Doppler ultrasonography are used routinely, being very useful in the evaluation of urologic obstruction or vascular pathologies [3]. When needed, CT scans, MRI and nuclear imaging techniques are also used [4]. Recently, reports are describing the use of contrast-enhanced ultrasonography (CEUS) as the diagnostic test for AAD.

This review intends to present an update of the use of CEUS as a diagnostic tool for AAD and differential diagnosis of different allograft pathologies.

\section{Invasive and noninvasive (non- ultrasonographic) morphological diagnosis of renal graft dysfunction}

\section{a. Kidney biopsy}

The morphological diagnosis of graft dysfunction can be made only by allograft biopsy, which is an invasive method. Non-invasive techniques like the US, CT, and MRI may have a complementary role.

After the exclusion of urologic and vascular causes of allograft dysfunction (AD), the workup protocols recommend having an allograft biopsy, a procedure with many possible complications such as bleeding and arterialvenous fistulae [5-8]. It is also time-consuming and has interobserver variabilities. The pathology evaluation of the allograft biopsy is made using the Banff classification, which evaluates the morphology of all renal compartments (glomerules, tubules, arteries and capillaries, and interstitium). The inflammatory involvement of the arteries, arterioles, and peritubular capillaries are a key target for the
CEUS evaluation. The acute modifications are characterized by cellular or humoral inflammatory pathway activation. The chronic modifications are represented by intimal hyperplasia, arteriolar hyalinization, and the splitting of the tubules basal membrane [9]. ATN and AHR have many common morphological aspects, such as the lesions of the tubular epithelial cells or the inflammation of peritubular cells. For the differentiation, immunochemistry staining is used. It is good to mention that the allograft biopsy is still the gold standard for the diagnosis of ATN and AHR [10,11].

b. Non-ultrasonographic, sectional, and functional imaging explorations

As mentioned above, there are other imaging evaluation methods used in the workup of AAD like CT scans, MRI, and single-photon emission computed tomography (SPECT).

The magnetic resonance imaging (MRI). MRI has the advantage of using no radiation and analyzing the morphology of the tissue in many sequences like T1 and T2 [12]. MRI can evaluate many aspects of the allograft kidney like renal perfusion, oxygenation, and inflammation [13]. MRI elastography can evaluate the fibrosis level of the allograft but with no information regarding its functional status [14]. There is no study published until now to evaluate the sensitivity and specificity of MRI in the diagnosis of AD. The lack of interest in the use of MRI in the workup of AD is because of the risk of Gadolinium renal toxicity which, in extreme cases, can lead to systemic nephrogenic fibrosis in a patient with renal insufficiency or chronic hemodialysis [15].

Computed Tomography (CT). CT scan is also used in the workup of $\mathrm{AD}$, but again, because of the nephrotoxicity of the contrast agent, it is less used [15].

Single-photon emission computed tomography (SPECT). It is a conventional nuclear imaging technique that is used for the assessment of the allograft function.

Positron emission tomography-computed tomography (PET-CT). It uses glucose analogs functionalized with radioisotopes in order to evaluate the cellular metabolism of different tissues. The technique is done in a "whole body" manner [16], and it has a sensitivity of $100 \%$ and specificity of $50 \%$ in the diagnosis of AR [17]. The disadvantage of the isotopic techniques is the irradiation, while the benefit is the high sensitivity and the operatorindependent interpretation.

\section{Ultrasonographic diagnosis}

Current ultrasonography (US) evaluation techniques can evaluate the morphology and vascular status of the allograft with the greyscale US, color Doppler and CEUS. These techniques are noninvasive, well tolerated by the patient, and can provide complex information about the status of the allograft, being also relatively reproducible. A great advantage for the use of the US is the position of the allograft in the iliac fossa that offers a perfect visualization of the kidney with no interference of other organs. 


\section{a. "Gray scale" ultrasonography}

The US provides morphological details of the allograft, such as position, form, and relationships with neighboring organs (Figure 1). It can differentiate the two components of the renal parenchyma (cortex and medulla), and it can evaluate the functionality of the allograft. US criteria for the diagnosis of $\mathrm{AD}$ are allograft enlargement, hyperechogenic cortex and hypoechogenic medulla with the unclear delimitation of the two structures [18-20]. In $\mathrm{AD}$, liquid collections may appear surrounding the allograft or the nearby organs, US being able to diagnose even small accumulation of liquid [21]. It is worth mentioning the possibility of the newest US machines to detect and characterize blood flow in medium to small vessels in the allograft.

There are two ultrasonographic vascular modes that use different technologies and principles: Doppler and CEUS. Both modes are presenting the "real-time" situation of the blood flow in a specific part of the allograft called the region of interest (ROI). Both techniques are operator dependent. The main differences regarding Doppler vs CEUS are the need for additional acquisition of the program and the need for a large band transducer (see Tables I and II).

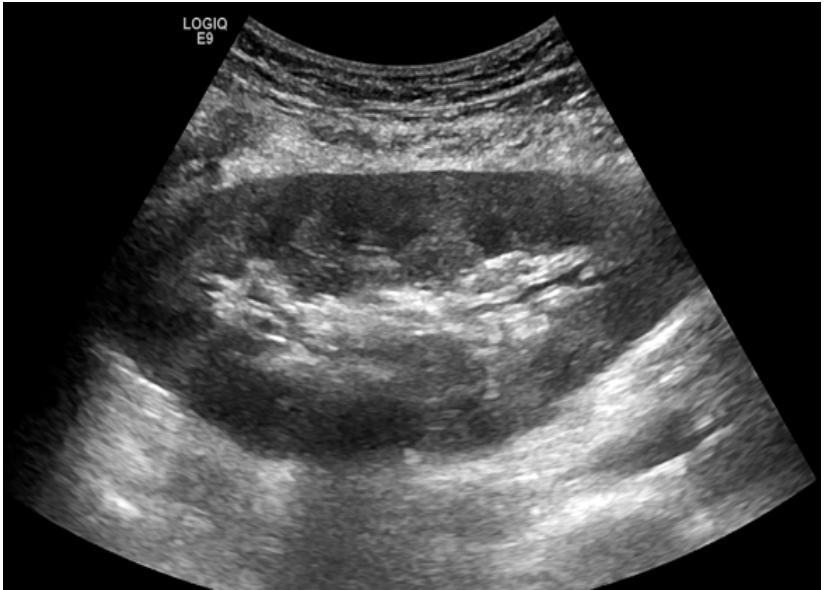

Figure 1. Grey-scale ultrasonographic appearance of a renal graft located in the right iliac fossa. The image quality is superior to the ultrasonographic appearance of the kidney in its native (lumbar) position due to the superficial position of the organ. The kidney appears as an oval structure where in the periphery there is the parenchyma (with more hypo-echogenic areas corresponding to the medulla) and in the center the hyper-echogenic appearance of the pyelocalyceal system.

Table I. Vascular explorations using the Doppler and CEUS ultrasound techniques. Similarities.

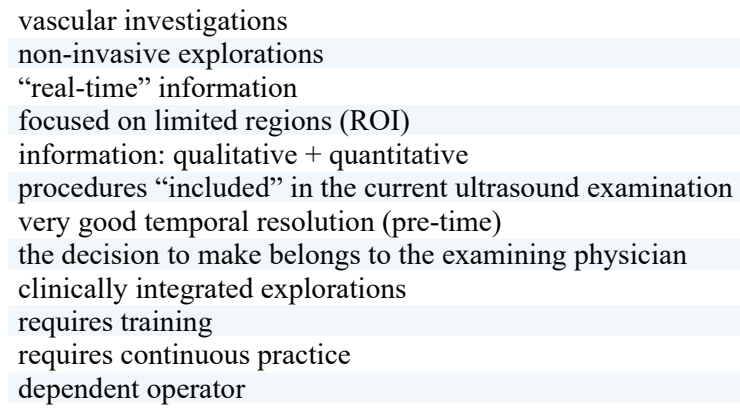

Table II. Vascular explorations using the Doppler and CEUS ultrasound techniques. Differences.

\begin{tabular}{|c|c|c|}
\hline Criteria & Doppler & CEUS \\
\hline Principles of the method & $\begin{array}{l}\text { vectorial information; } \\
\text { shows the flow speed and blood flow direction; } \\
\text { the source of the echoes - groups of red blood cells; } \\
\text { delivered ultrasound information - specific located (Doppler } \\
\text { sample positioned in the middle of the vessel) }\end{array}$ & $\begin{array}{l}\text { harmonic information } \\
\text { evaluation of the contrast agent transit through } \\
\text { the circulatory bed } \\
\text { echoes types: harmonic } \\
\text { echoes source - microbubbles information } \\
\text { delivered - regional (all field) }\end{array}$ \\
\hline Sensitivity & limited to $2 \mathrm{~cm} / \mathrm{sec}$ & independent of blood movement \\
\hline Technology & integrated equipment & $\begin{array}{l}\text { additional } \\
\text { soft }+\mathrm{AC}+\text { broadband transducer }\end{array}$ \\
\hline $\begin{array}{l}\text { The error factors (related } \\
\text { method) }\end{array}$ & $\begin{array}{l}\text { insonation angle }(<60 \text { degrees }) \\
\text { the size of the sample collection area } \\
\text { PRF }\end{array}$ & $\begin{array}{l}\text { attenuation } \\
\text { the number and position of the outbreak } \\
\text { IM }\end{array}$ \\
\hline Vessels explored & $\begin{array}{l}\text { arteries and veins } \\
\text { medium and large trunks } \\
\text { microcirculation: indirect (IP. IR, S/D) }\end{array}$ & $\begin{array}{l}\text { arteries } \\
\text { medium and large trunks } \\
\text { microcirculation }\end{array}$ \\
\hline Information obtained & $\begin{array}{l}\text { speed }(\mathrm{cm} / \mathrm{sec}) \\
\text { flow type (pulsatility); } \\
\text { sense of movement } \\
\text { movement pattern (laminar, turbulent); }\end{array}$ & $\begin{array}{l}\text { characteristics of blood transit through ROI } \\
\text { the total and partial flow duration (sec) } \\
\text { microvascular exploration }\end{array}$ \\
\hline $\begin{array}{l}\text { Dependence of the quality } \\
\text { image in grey scale }\end{array}$ & partial & total \\
\hline Dependence on depth & partial & partial \\
\hline
\end{tabular}




\section{b. Doppler vascular ultrasonography}

Due to the superficial position of the renal graft in the iliac fossa, as well as the easy visualization of the anastomosis of the renal vessels with the iliac vessels, Doppler ultrasound is widely used in the kidney transplant, being accessible both in the operating room and at the patient's bed.

It helps in early diagnosis of large and medium vessel complications, allowing reintervention and thus the saving of the renal graft [22]. Therefore events like renal artery and vein thrombosis, renal artery stenosis, renal infarcts, aneurysms, and arteriovenous fistula are frequently and easily diagnosed thanks to Doppler ultrasound [3].

On the other hand, the diagnosis of acute and chronic rejection involves pathological changes, that affect peritubular and glomerular capillaries, type of vessels at which Doppler ultrasound cannot quantify directly hemodynamic parameters [23].

Doppler mode can be color and power, offering valuable information regarding intrarenal vasculature. Color Doppler mode can present the vascular status of a certain area, whereas power Doppler mode is focused on the single blood vessel, these two techniques being complementary (Figure 2). The Doppler mode offers information regarding the presence of blood flow, distribution, pulsatility, and amplitude of the allograft vasculature. Also, it can measure the blood flow speed in $\mathrm{cm} / \mathrm{sec}$. The ratio between systolic and diastolic velocities is called impedance indices (resistivity - RI; pulsatility - PI; systolic diastolic ratio $\mathrm{S} / \mathrm{D}$ ) and correlates with the quality of the parenchymal circulatory bed [24].

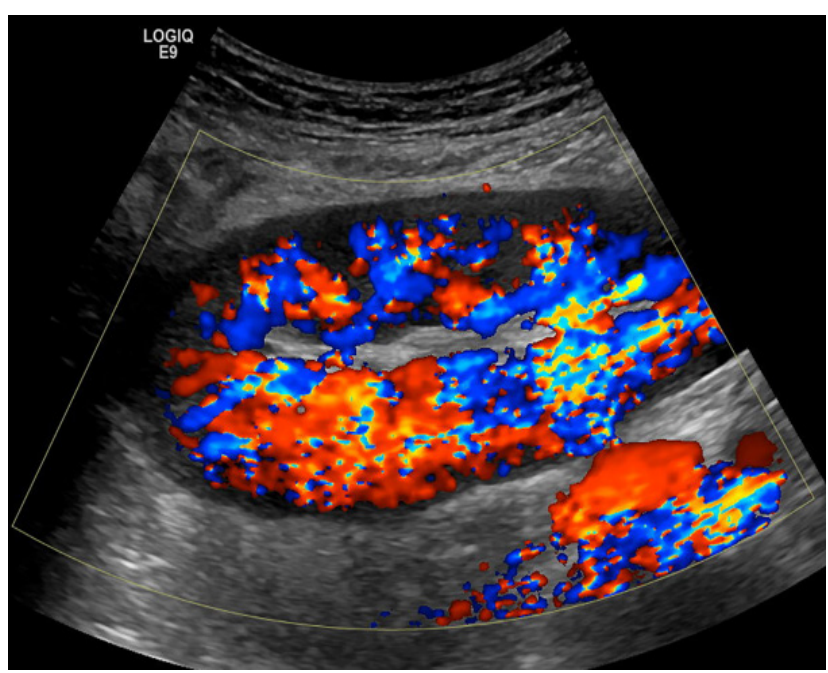

Figure 2. Ultrasonographic appearance in color Doppler mode of a transplanted kidney. The presence of the circulatory signal starting from the renal hilum and ending with the arch arteries, in subcapsular position, is noticed. The red and blue coloring of the signal is a convention; the color being directly correlated with the direction of movement of the blood column relative to the transducer.
Impedance indices are measured at the level of the interlobular and arched arteries representing an indirect assessment of renal microcirculation (Figure 3). Cano et al. have shown that RI is a useful marker in evaluating posttransplant renal function, so that an increase of more than 0.7 raises the suspicion of acute renal graft dysfunction, but without distinguishing between AR, ATN, toxicity to inhibitors calcineurin, renal vein thrombosis, ureteral obstruction or acute pyelonephritis [25].

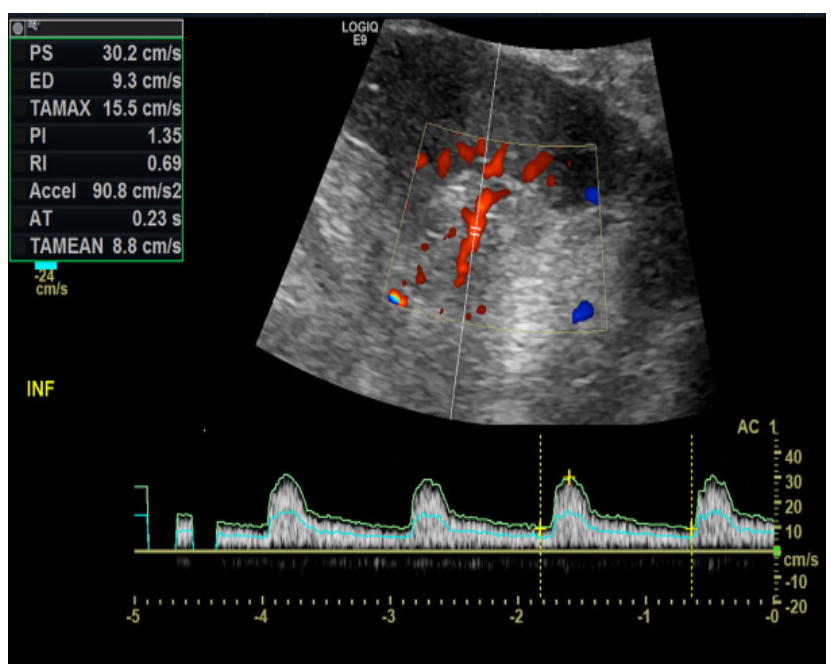

Figure 3. Spectral Doppler ultrasound aspect of a blood flow passing through main artery of a kidney graft. The systolicdiastolic waveform may be noticed - where the minimum speed is upper to the 0 line - suggesting a low resistance circulatory bed. The laminar flow can be seen by placing the velocity vectors in the upper area of the curve. The distribution of vectors above line 0 suggests oriented flow transducer.

Several researchers have shown that RI and PI measured immediately after transplantation predict longterm renal graft outcome [26-29]. More than that, the vascular resistivity index measured by Doppler ultrasound is correlated with the resistivity index recorded by the life-port perfusion machine used for kidney preservation. It may predict the primary graft outcome [30]. Seiler et al. measured resistivity index in different organs of the transplanted patient, including the renal graft, and concluded that RI indicates the presence of systemic vascular pathology, predicting mortality rather than renal graft dysfunction [31], being affected by pulse pressure, frequency and heart rate [32].

In the same manner, Heine and co-workers found an increased RI associated with subclinical systemic atherosclerosis, the pulse pressure, age over 60 years, female sex, and the presence of diabetes. They did not find any correlation between RI and GFR or RI and the age of the donor [33]. 


\section{c. Harmonic vascular ultrasonography using i.v. contrast agents - CEUS}

Initially, CEUS was used in liver pathology, later proving its usefulness in the evaluation of renal graft. The elements that recommend the exploration of CEUS in renal graft pathology are the superficial position of the graft, the lack of irradiation and the toxicity of the contrast substance, the repeatability of the method, as well as the ease of performing the method at the patient's bed. The contrast agent currently used in the EU is called SonoVue ${ }^{\circledR}$ (Bracco, Italy). It contains sulfur hexafluoride functionalized microbubbles having a phospholipid membrane (eliminated predominantly by the respiratory tract) that are small enough (about $2.5 \mu \mathrm{m}$ ) to penetrate the renal microcirculation without subsequently entering the renal interstitium. The administration of SonoVue has minimal risk to the patient $[34,35]$. SonoVue ${ }^{\circledR}$ is insoluble in water or body fluids so that once it reaches the blood, it reflects the ultrasound (the acoustic impedance of the microbubbles is about $25 \mathrm{~dB}$ greater than that of the blood) in the form of harmonic echoes, giving it a more hyper-echogenic appearance compared to that of adjacent tissues [36]. The microbubbles break when using a mechanical index that is too high (usually $>0.5$ ). Therefore a mechanical index of 0.1 or less is required. After the intravenous administration of SonoVue, the procedure is recorded for 3-4 minutes.

The analysis of the CEUS procedure has three components: observational, quantitative, and semiquantitative.

1. Observational analysis. It consists of dynamic exploration using two ultrasonographic fields (one that gives fundamental echoes, another that gives harmonic echoes) and the evaluation of the loading times of the circulating bed for the total duration of the exploration. After administration of the contrast substance, three phases can be identified:

a. early arterial phase in which the arteries are visualized, observing the progression of the contrast substance at the level of the iliac artery, the renal artery, the interlobular artery, the interlobular arteries, and then the arched arteries (first 20 seconds);

b. delayed arterial phase (or cortical phase) - lasts approx. 20-40 seconds and in this phase the contrast agent is uniformly captured in the renal cortex with a clear cortico-medullary differentiation;

c. the medullary phase (also called nephrographic) can be followed for 40-120 sec after the injection of the contrast substance. In this phase, there is a gradual loading of the contrast agent at the level of the Malpighi pyramids until the cortex and medulla have equal amounts of contrast agent. The disappearance of the contrast agent from the kidney usually occurs after 2 minutes, but sometimes it can persist for up to 4-5 minutes (Figures $4 \mathrm{a}, 4 \mathrm{~b}$ and $4 \mathrm{c}$ ).

2. Quantitative analysis. The quantitative CEUS exploration is a distinct component, obtained by refining stored temporal information. The analysis is focused on the dynamics of the transit of the contrast agent through ROI and aims to measure perfusion parameters related to the circulatory bed. Besides the advantage of representing certain hemodynamic phenomena through graphical information ("time-intensity curves"), it also allows their numerical analysis in comparison with other regions considered by reference (normal or with different types of pathologies).

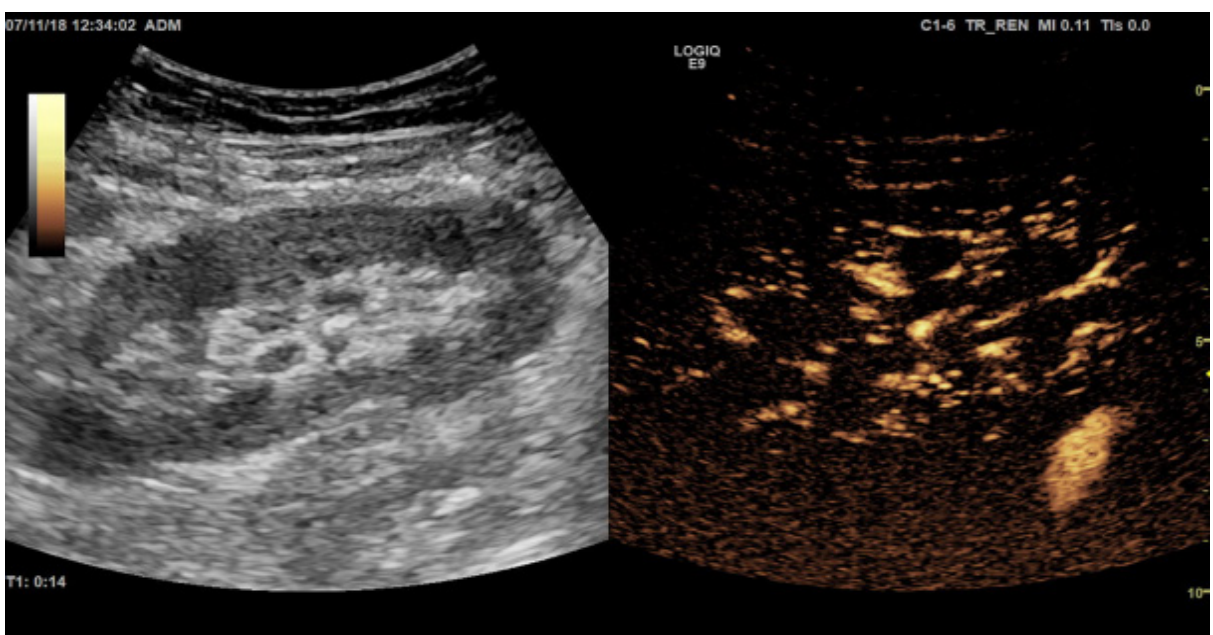

Figure 4a. Early arterial phase $(<20 \mathrm{sec})$. On the left: the image created by fundamental echoes. On the right: the harmonic, vascular image. To notice the enhance at the level of the segmental arteries followed by the arcuate arteries. 


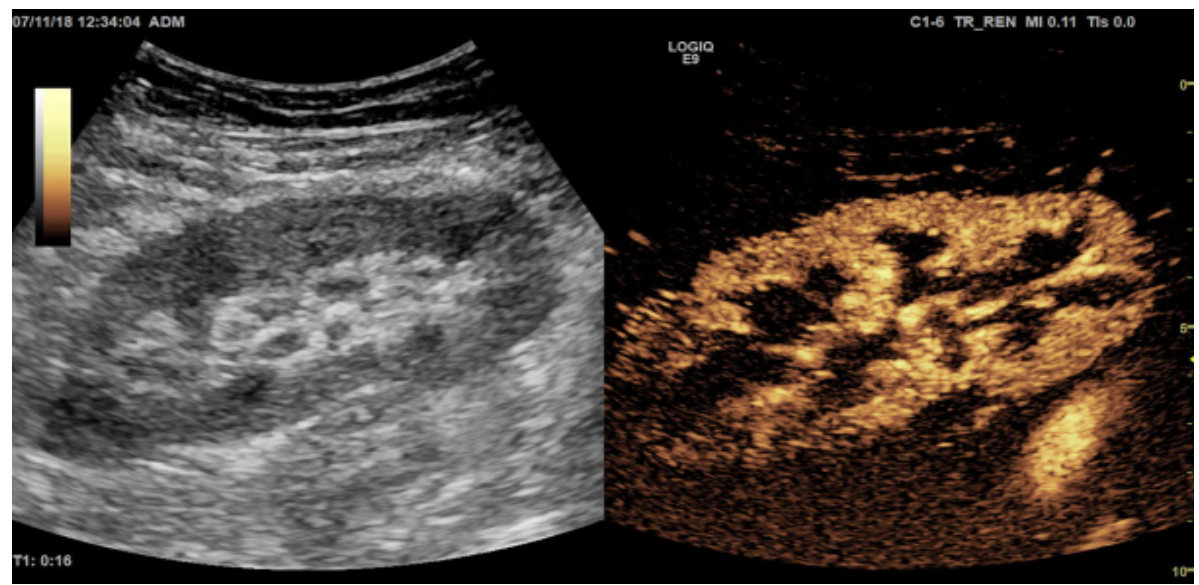

Figure 4b. Delayed arterial phase (or cortical phase) - lasts approx. 20 - 40 seconds. On the right image it is noticed the full enhancement of the cortical parenchyma; Accentuated hypoechoic aspect of the medulla may suggest the wrong appearance of renal cyst.

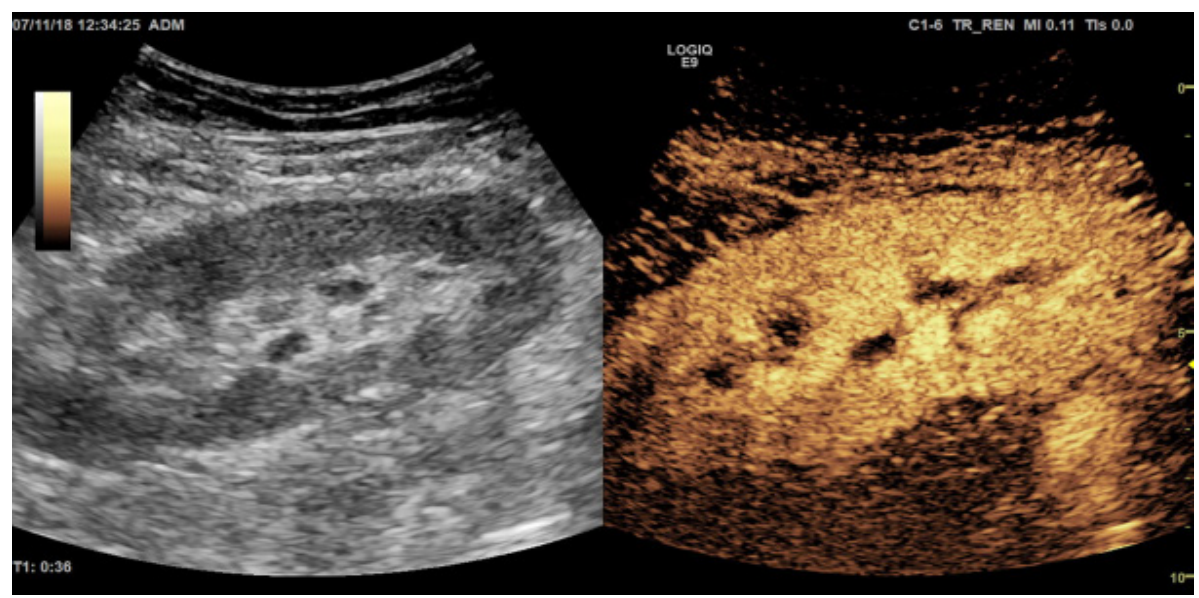

Figure 4c. The medullary phase (also called nephrographic) can be followed for $40-120 \mathrm{sec}$ after the contrast administration. In this phase, the contrast agent is uniformly captured in the renal parenchyma.

The visual representation of the variation of the signal intensity over time is called the "time-intensity curve" (TIC). There is a direct correlation between the characteristics of the curve and the peculiarities of the circulating bed. By applying a mathematical model that describes the curves, one can obtain numerical information called TIC parameters, such as: the contrast agent transit rate through ROI (WiR "wash in rates"); peak height (PE - "peak enhancement"), area under the curve (WiAUC - "area under the curve during wash in"), peak time (TTP - "time to peak"), rise time (RT - "rise time") or the average transit time (mTTl - "medium transit time, local"). Measurements are made either after the bolus injection of the contrast agent (non-cardiac applications, only once) or after continuous administration of it in the form of infusion (cardiologic applications, several times, each measurement being preceded by the bursting of the bubbles generated by the increase of IM at high values, over 0.5). The limits of the procedure are represented by the variability of the harmonic signals, depending on the equipment used and not the standardization of the information collection mode. The advantages of the method are good accuracy, noninvasiveness and lack of irradiation, possibility of repetition whenever necessary, relatively low costs and reproducibility. The large number of parameters that can be studied makes this application a valuable tool for research in various medical areas [37].

The TIC analysis can be performed after the patient has left the medical office, being performed offline. The curves are generated at the level of samples (round, rectangular, or "freehand") set by the examiner related to ROI from different areas of the kidney. At the level of each sample, the ultrasonographic equipment will represent on the screen functions of the signal intensity variation (in $\mathrm{dB}$ or acoustic units - a.u.) over time. Following the calculations made by the program, the time of entry and the total duration of the contrast agent transit through ROI, the time of systolic ascension, the time of unloading, the area under the curve will be recorded (Figure 5). 


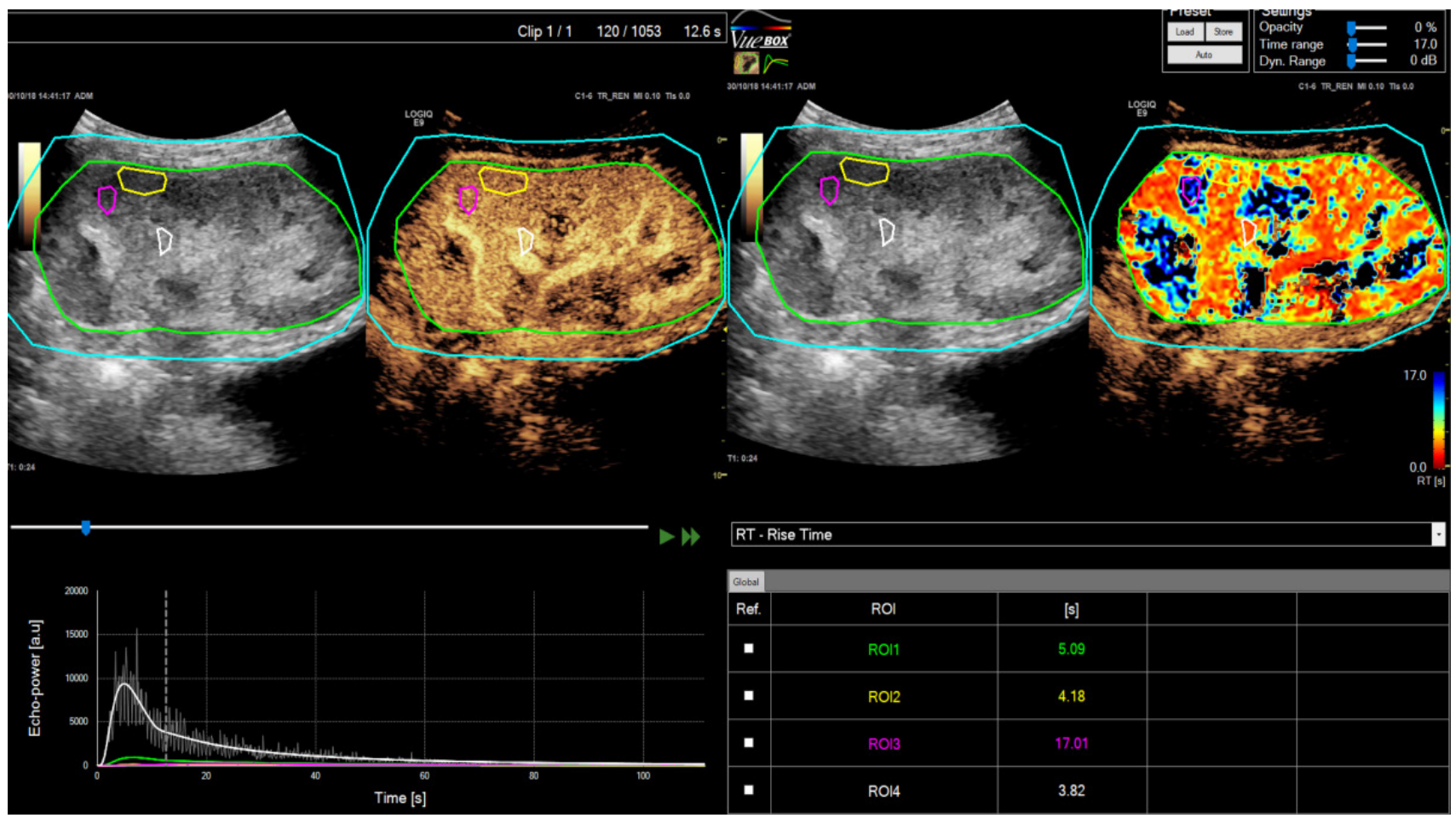

Figure 5. Quantitative analysis of the contrast passage mode in different anatomical regions at of the renal graft.

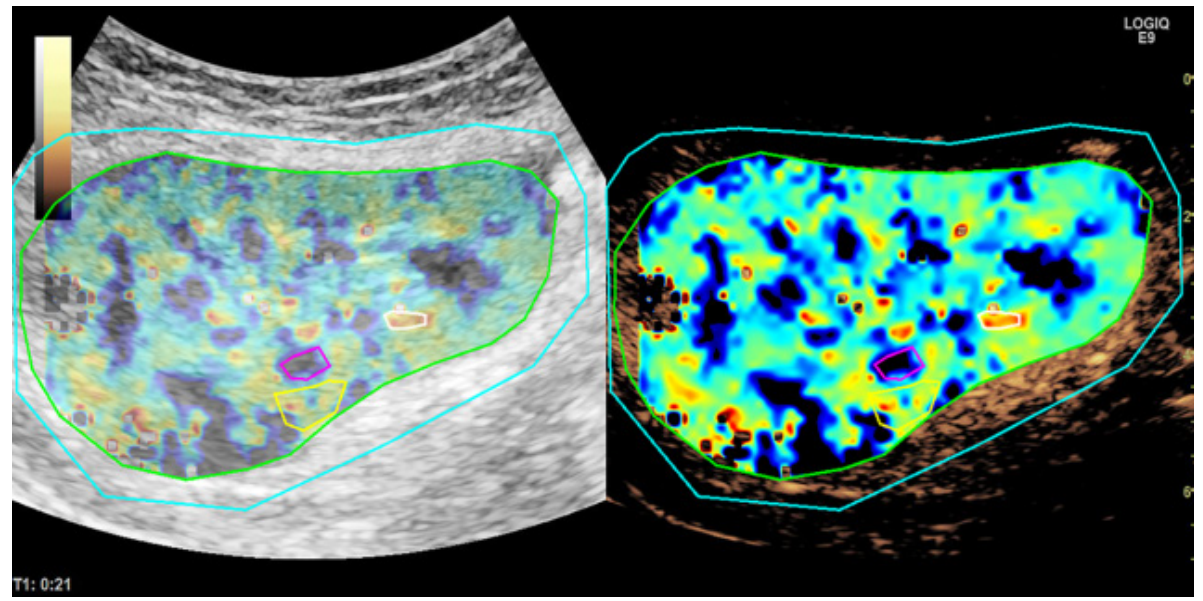

Figure 6. Parametric, semi-quantitative analysis of the contrast passage mode at the level of the circulatory bed in a limited time. Each moment of penetration of the contrast agent has a different color representation.

3. Semi-quantitative analysis. The semi-quantitative analysis consists of the superimposition of a color map over the ultrasound image, through which temporal information from a whole contrasting examination loop is connected. The analysis allows the assessment of a static image of some dynamic elements, such as the direction and the speed of capture in different regions of the image. It is necessary to select a time duration for the moment when the contrast agent becomes visible in the circulatory bed of the graft and to select the "parametric" application. The equipment will automatically represent in different colors the sequence of filling the circulatory bed (Figure 6).

The guide for non-hepatic applications of the European Federation of Ultrasonography Societies in Medicine and Biology (EFSUMB) 2017 recommends the use of CEUS in renal transplantation for vascular and ischemic complications of the renal graft, characterization of complex cysts - according to the Bosniak classification, in undetermined renal injury, as well as and in acute pyelonephritis. The guide concludes that quantitative 
functional information resulting from the use of the TIC curve may reveal altered renal microperfusion, but requires further investigation in large batches of patients in order to understand the importance of these changes in transplanted kidney pathology [38].

Intravenous contrast harmonic ultrasonography in the evaluation of the renal graft function

a. CEUS parameters correlate with renal

The CEUS parameters are correlated with the renal function, a fact initially demonstrated by the Italian team formed by Farina and collaborators. They enrolled 56 kidney transplant patients in the study, explored by CEUS using a 1st generation Levovist contrast agent, and noted that slow loading time and delayed washout were associated with graft dysfunction and reduced renal function. At the same time, they observed an inversely proportional variation of the TTP values depending on the creatinine clearance $(\mathrm{ClCr})$. Thus in the group with chronic rejection, there was a $\mathrm{ClCr}$ of approx. $28-42 \mathrm{ml} / \mathrm{min}$ and a TTP $=235$ sec. In the group with ATN a $\mathrm{ClCr}=32-60 \mathrm{ml} / \mathrm{min} \mathrm{TTP}=130$ sec was found. At the same time the control group had a $\mathrm{ClCr}=92-112 \mathrm{ml} / \mathrm{min}$ and TTP $<65 \mathrm{sec}$. [39]. Wang and co-workers have shown that CEUS examination expresses quite precisely the microcirculatory characteristics of renal parenchyma, and a correlation between the value of the parameters of this examination and the renal function can be established. The grafts that had dysfunction required a longer loading time with the contrast substance both in the renal cortex and in the renal medulla. There were statistically significant differences between the group with normal and abnormal kidney function transplanted with lower values of the slope of the ascending cortex at the cortical level (A1 - 1.27 \pm 0.57$)$ and that at the medullary

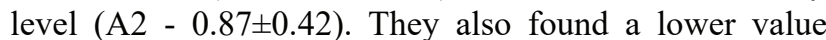
of maximal intensity (DPI1 - 7.45 \pm 3.38 ) in the group of patients with graft dysfunction versus the group without dysfunction (A1 - $1.79 \pm 0.59 \mathrm{sec}$; A2 - $1.24 \pm 0.50 \mathrm{sec}$ DPI1 - $9.75 \pm 1.65 \mathrm{sec})[40]$.

\section{b. CEUS examination identifies early AD microcirculatory changes}

Renal biopsy and histopathological examination led to a better understanding of the pathophysiology of these entities, but this method is burdened with numerous risks. Over time, Doppler ultrasound was introduced, which, using indirect evaluation like spectral Doppler with the help of RI, PI and S/D parameters of microcirculation resistance, could raise the suspicion of AAD due to the immunological cause. More recent studies have shown, however, that the impedance indices mentioned have good sensitivity but low specificity [25]. Under these conditions, there was a need to find new diagnostic methods with minimal risks for both the patient and the kidney graft. Currently, the use of CEUS in renal transplantation refers to the urological pathology of renal graft [38]. However, its role in nephrological pathology (AR, ATN) is a promising one [41], the general desire being to develop diagnostic models based on micro vascularization of the renal graft to avoid unnecessary biopsies of the transplanted kidney. A first study addressing the value of CEUS examination in the immediate posttransplant period was conducted by Fischer et al. in 2004 on 18 patients, noting that the quantitative parameters used in contrast ultrasound can identify early acute rejection prior to RI modification. Patients were evaluated with the US in both B and Doppler mode, respectively CEUS, at 5 and 6 days post-transplant. The authors demonstrated that in the rejection group (6 patients), the TIC curve revealed a delay in the progression of the contrast substance in the subcapsular area (TTP of $32.9 \pm 8.3 \mathrm{sec}$ ) and a less pronounced cortical intensity $(3.8 \pm 2.3 \mu \mathrm{l} / \mathrm{sec})$ compared to that of the renal artery $(7.9 \pm 50.9 \mu 1 / \mathrm{sec})$ and interlobular arteries $(8.7 \pm 3.8 \mu \mathrm{l} / \mathrm{sec})$ respectively. On the other hand, in the non-rejection group ( 6 patients) the TIC curve had a rapid and abrupt increase (TTP $20.9 \pm 4.7 \mathrm{sec}$ ). The intensity of the harmonic signal of the contrast substance was similar in the renal artery $(11.7 \pm 4.5 \mathrm{iu} / \mathrm{sec})$, the interlobular artery $(8.7 \pm 4.6 \mathrm{iu} / \mathrm{sec})$ and the subcapsular region $(8.3 \pm 3.7 \mathrm{iu} / \mathrm{sec})$ [42] (Figures 7 and 8).

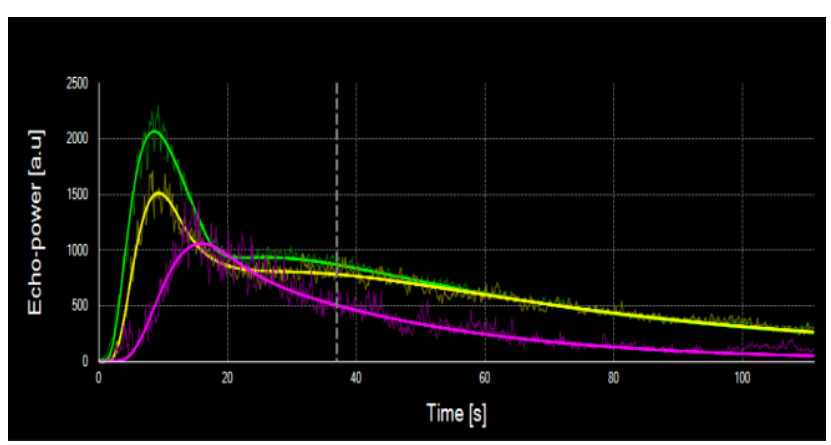

Figure 7. Time intensity curve (TIC) in case of acute rejection renal parenchyma. A delay in the wash-in and wash-out can be observed.

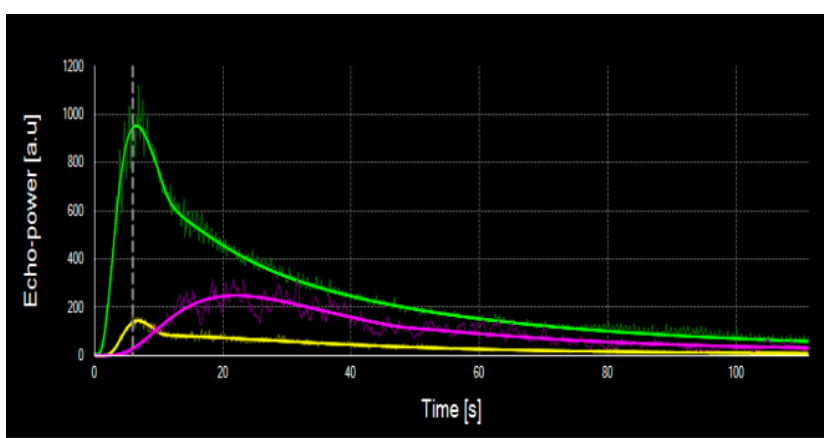

Figure 8. Time intensity curve (TIC) in case of normal renal graft parenchyma. A fast wash-in and wash-out can be observed (the outflow slope is "sharp"). 


\section{c. CEUS allows discrimination between graft dysfunction and normal renal function}

Grzelak et al. performed a study on 63 patients ( 35 with stable renal function and 28 with AAD) and analyzed CEUS and Doppler parameters at 70-120 h post-transplant. They found that AAD patients had a statistically significant delay in the progression of the contrast substance in the areas of interest. The mean contrast agent uptake time in the renal cortex versus the segmental arteries was $2.35 \pm 0.69 \mathrm{~s}$ in RA, $2.08 \pm 0.69 \mathrm{~s}$ in ATN, respectively $0.92 \pm 0.53 \mathrm{~s}$ in the group with stable renal function. At the level of the renal pyramids, the mean contrast agent uptake time was $3.25 \pm 0.81 \mathrm{~s}$ in RA; $3.03 \pm 0.81 \mathrm{~s}$ in ATN; respectively $1.2 \pm 0.62 \mathrm{~s}$ in the group with stable renal function. The use of CEUS better distinguishes the group of patients with renal dysfunction from that of normal function, compared with the use of impedance indices (RI, PI, S/D) in Doppler ultrasound. A delay of more than 2 seconds in the capture of the contrast substance strongly suggests a renal graft dysfunction [43].

\section{d. CEUS examination allows an etiological diagnosis of graft dysfunction}

The lack of clinical signs or specific laboratory values makes the differential diagnosis between ATN and RA more difficult. At present, the differentiation can be done only with the help of histopathological examination, the development of new diagnostic models based on the evaluation of renal microcirculation being desirable.

Fischer et al. re-evaluated the efficiency of the contrast ultrasound technique in a batch of 32 patients during the first 5-7 days after transplantation. Patients were divided into 3 subgroups: acute rejection (n-7); perirenal hematoma (n-6); non-rejection (with retained renal function, $\mathrm{n}$-17). It was observed that in the group of patients with a perirenal hematoma the loading with the contrast substance was heterogeneous, helping to delimit the hematoma, but its intensity in the renal cortex $(9.2 \pm 3.8 \mathrm{~dB} / \mathrm{sec})$ was lower compared to that of the renal artery $(15.3 \pm 6.3 \mathrm{~dB} / \mathrm{sec})$ [44].

In the patients with rejection the intensity of contrast in the renal cortex $(7.4 \pm 3.2 \mathrm{~dB} / \mathrm{sec}$ vs. $10.3 \pm 4.3$ $\mathrm{dB} / \mathrm{sec}$ in the non-rejection group) was lower than in the renal artery $(10.7 \pm 4.3 \mathrm{~dB} / \mathrm{sec}$ vs. $12.5 \pm 5.5 \mathrm{~dB} / \mathrm{sec})$, and the temporal difference seen graphically between the subcortical area and the renal artery was greater in the rejection group $(\Delta \mathrm{t}=2.27 \pm 0.73 \mathrm{sec})$ versus the non-rejection group $(\Delta \mathrm{t}=0.97 \pm 0.62 \mathrm{sec})[44]$.

The same group of researchers led by Thomas Fischer evaluated 48 patients at 4-10 days after transplant. The authors defined the "infusion coefficient" (PQ) as the ratio of filling time in the interlobular artery and the renal cortex. This parameter showed double values in the vascular rejection group $(n=5)(2.2 \pm 0.8)$ compared to the control group (1.2 \pm 0.4$)(\mathrm{n}=19)$. At the same time, patients with $\operatorname{ATN}(n=7)$ and those with perirenal hematoma $(n=7)$ had higher values of the infusion coefficient $(1.6 \pm 0.7)$, respectively (1.7 \pm 0.4$)$ compared to the control group. Patients with non-vascular rejection (7) had similar TIC curve values as those in the control group [45].

Fischer and colleagues subsequently tried to develop a standardized tool for the diagnosis of acute rejection. The study included 12 patients in the nonrejection group, 8 patients with rejection, and 2 patients with perirenal hematoma. The time elapsed until the first increase in signal intensity in the renal artery and the renal cortex was calculated, determining the difference between the two values. This time difference was higher in the rejection group $(2.2 \pm 0.7 \mathrm{sec})$ than in the control group $(\mathrm{p}<0.05)$. Also, as in the first studies, an increase in the time difference was also identified in the two patients who had a perirenal hematoma. Patients were examined at 6 days post-transplant both with contrast and conventional ultrasonography, measuring volume and impedance indices (RI, PI, S/D) giving uncertain results [46].

Benozzi and colleagues performed a study on 39 patients in the first 30 days after transplantation. They were examined in Doppler mode and by CEUS technique. With the help of the first ultrasound method, the increase of the resistivity indices led to the identification of the patients with AAD but did not provide information to differentiate the AR from the ATN. When examining by CEUS, an increased value of TTP was observed in the group of patients with AR compared to those in the control group. Also, in patients with ATN, the infusion ratio between medullary and cortical (RBV) was $<0.81$ and the mTT (mean transit time) between medullary and cortical was $<0.87$, both values lower than those of the control group [47].

The usefulness of the CEUS method in the diagnosis of RA has been demonstrated by a group of Chinese researchers by describing a high sensitivity and specificity. They enrolled 57 patients in the study who were followed for an average of 20 months after transplantation. A number of indicators were identified: RT and TTP at the medullary level (RTm/TTPm) and the interlobular artery (RTi/TTPi), as well as the difference of the same parameters between medullary and cortical ( $\Delta$ RTm-c and $\Delta$ TTPm-c), which were statistically significantly higher in patients with acute rejection than in the control group. In addition, RTm, TTPm, $\Delta \mathrm{RTm}$-c, and $\Delta$ TTPm-c were significantly higher in AR patients than in ATN patients. The IR level in patients with ATN was higher than in the healthy ones, but without any increase in the acute rejection group [48].

Regarding the differential diagnosis of $\mathrm{AD}$, Fernandez and collaborators performed a study on five patients who subsequently required emergency nephrectomy in which the histopathological diagnosis was acute cortical necrosis. They showed renal artery loading followed by renal pyramidal loading, with a defect in the cortical banding, which is comparable to that identified in CT and MRI [49]. 


\section{e. The exploration of CEUS allows anticipating} the evolution of the renal function in the transplanted patient

This will help thenephrologist to morecloselymonitor and individualize the immunosuppressive therapeutic plan in patients at risk. The vigilant attitude translates into early treatment and an increase in the survival of the renal graft. Kay et al. conducted a pilot study that aimed to specify the prognostic value of contrast substance use on ultrasound in the immediate post-transplant period, proving that CEUS is reproducible and has prognostic value. Thus the "area under the curve" determined in the first week after transplantation was correlated with renal function at three months [50]. The prognostic value of CEUS was also demonstrated by Schwenger et al., who evaluated CEUS and Doppler at seven days and one year after transplantation in a group of 68 patients. It has been observed that the values of renal blood flow measured with the help of CEUS in the first week after transplantation correlates with the renal function at one year and with the age of the donor, and among the values of the histopathological scores corresponding to the Banff classification are correlated with the intimal fibrous thickening. Also, the resistivity index was correlated with the age and the vascular compliance of the recipient [51].

Mori et al. performed research on a group of 39 transplanted patients that he followed for four years, observing a decrease in the rate of glomerular filtration in the first year but maintaining the same values for the next 3 years. CEUS parameters represented by mean transit time (MTT), cortical blood flow (cRBF) and regional blood volume (RBV) were positively correlated with glomerular filtration rate and medullary blood flow (mRBF) was negatively correlated. A good cortical renal perfusion immediately after transplant can predict a favorable evolution of the graft in the following years. It was concluded that the Doppler examination has a high sensitivity in terms of graft dysfunction, and CEUS has increased specificity in relation to the renal parenchyma infusion rate, the two examinations combined providing promising long-term prospects [52].

A recent study, conducted by Stenberg, has managed to identify, using CEUS, infusion defects of up to $0.2 \%$ of total renal volume, which have been detected in a large number of patients (20 out of 99 patients). The extent of these non-perfused regions correlated with renal function at 1 month and 3 months post-transplant [53].

f. CEUS examination is superimposed on renal graft biopsy in well-defined situations

Currently, researchers are looking for ways to replace biopsy by numerous non-invasive diagnostic procedures. In this regard, Muller-Peltzer et al. tried to analyze the parameters of the diagnostic test for CEUS compared with renal biopsy. They noted a sensitivity of $85.7 \%$, specificity of $100 \%$, a positive predictive value of $100 \%$ and a negative predictive value of $98 \%$. The study analyzed 57 patients with impaired renal function, of whom 7 had acute vascular rejection. CEUS was able to identify 6 of the 7 cases, but could not differentiate the histological subtypes of rejection (cellular versus humoral) [54].

\section{Conclusions}

After proving its diagnostic qualities in liver pathology [55], CEUS extends its applicability to other parenchymal organs as well, the transplanted kidney being one of the most recently studied organs with this technique. Different specialized centers evaluated the characteristic parameters of CEUS at the level of renal graft, comparing the results obtained with those of other rejection diagnostic techniques. Most studies have shown a delay in capturing the contrast agent and a lower intensity in the areas of interest in both acute graft dysfunction, acute and chronic rejection, acute tubular necrosis, and large perirenal hematoma.

Compression of hematoma or interstitial edema from acute tubular necrosis on the vascular bed could explain the changes observed with the help of CEUS in these pathologies. The reduction of cortical perfusion, evidenced by the CEUS technique, would indicate the reduction of GFR [52] and the presence of acute rejection [44]. These data are in accordance with the study of Sis et al. [56] who demonstrated that lesions specific to acute rejection initially occur in the renal cortical area. At the current level of knowledge, CEUS is useful in the evaluation of renal micro vascularization, being able to identify small defects up to 0.02 of the total renal volume [53] which could not be detected with the help of the Doppler. Also, a good renal infusion discovered immediately after transplantation can predict a favorable evolution of the graft in the long term [52]. However, in order to develop diagnostic models based on renal graft micro vascularization, studies are needed on larger groups of patients. It is important to note that chronic changes like fibrosis, intimal arterial hyperplasia, and glomerulosclerosis can cause alterations in renal micro vascularization. Probably, in the future, together with CEUS, other methods of ultrasound evaluation, such as elastography, will contribute to increasing the specificity of the diagnosis of renal graft pathologies. At present, CEUS cannot differentiate between the cellular and humoral rejection [45,54]. The histopathological examination is still the gold standard of diagnosis. CEUS has also proven its effectiveness in identifying vascular rejection, a pathological entity diagnosed with the help of this imaging technique by several teams of researchers $[45,46,54]$.

\section{Acknowledgments}

This work was supported by a grant of Ministery of Research and Innovation, CNCSIS - UEFISCDI, project number PN-III-P4-ID-PCE-2016-0701, PNCDI III contract number: 184 / 2017. 


\section{References}

1. Lim WH, Johnson DW, Teixeira-Pinto A, Wong G. Association between duration of delayed graft function, acute rejection, and allograft outcome after deceased donor kidney transplantation. Transplantation. 2019;103:412-419.

2. Kidney Disease: Improving Global Outcomes (KDIGO) Transplant Work Group. KDIGO clinical practice guideline for the care of kidney transplant recipients. Kidney Disease: Improving GlobalOutcomes (KDIGO) Transplant Work Group. Am J Transplant. 2009; 9 Suppl 3:S1-S155.

3. Granata A, Clementi S, Londrino F, Romano G, Veroux M, Fiorini F, et al. Renal transplant vascular complications: the role of Doppler ultrasound. J Ultrasound. 2014;18:101-107.

4. Köhnke R, Kentrup D, Schütte-Nütgen K, Schäfers M, Schnöckel U, Hoerr V, et al. Update on imaging-based diagnosis of acute renal allograft rejection. Am J Nucl Med Mol Imaging. 2019;9:110-126.

5. Redfield RR, McCune KR, Rao A, Sadowski E, Hanson M, Kolterman AJ, et al. Nature, timing, and severity of complications from ultrasound-guided percutaneous renal transplant biopsy. Transpl Int. 2016;29:167-172.

6. Reschen ME, Mazzella A, Sharples E. A retrospective analysis of the utility and safety of kidney transplant biopsies by nephrology trainees and consultants. Ann Med Surg (Lond). 2018;28:6-10.

7. Tapia-Canelas C, Zometa R, López-Oliva MO, Jiménez C, Rivas B, Escuin F, et al. Complications associated with renal graft biopsy in transplant patients. Nefrologia. 2014;34:115-119.

8. Tsai SF, Chen CH, Shu KH, Cheng CH, Yu TM, Chuang YW, et al. Current safety of renal allograft biopsy with indication in adult recipients: an observational study. Medicine (Baltimore). 2016;95:e2816.

9. Haas M, Loupy A, Lefaucheur C, Roufosse C, Glotz D, Seron D, et al. The Banff 2017 Kidney Meeting Report: Revised diagnostic criteria for chronic active T cell-mediated rejection, antibody-mediated rejection, and prospects for integrative endpoints for next-generation clinical trials. Am J Transplant. 2018;18:293-307.

10. Williams WW, Taheri D, Tolkoff-Rubin N, Colvin RB. Clinical role of the renal transplant biopsy. Nat Rev Nephrol. 2012;8:110-121.

11. Broecker V, Mengel M. The significance of histological diagnosis in renal allograft biopsies in 2014. Transpl Int. 2015;28:136-143.

12. Zhang JL, Morrell G, Rusinek H, Sigmund EE, Chandarana $\mathrm{H}$, Lerman LO, et al. New magnetic resonance imaging methods in nephrology. Kidney Int. 2014;85:768-778.

13. van Eijs MJM, van Zuilen AD, de Boer A, Froeling M, Nguyen TQ, Joles JA, et al. Innovative perspective: gadolinium-free magnetic resonance imaging in longterm follow-up after kidney transplantation. Front Physiol. 2017;8:296. doi: 10.3389/fphys.2017.00296.

14. Sarvazyan A, Hall TJ, Urban MW, Fatemi M, Aglyamov SR, Garra BS. An overview of elastography - an emerging branch of medical imaging. Curr Med Imaging Rev. 2011;7:255-282.

15. Hanssen O, Erpicum P, Lovinfosse P, Meunier P, Weekers L, Tshibanda L, et al. Non-invasive approaches in the diagnosis of acute rejection in kidney transplant recipients. Part I. In vivo imaging methods. Clin Kidney J. 2017;10:97-105.

16. Pauwels EK, Ribeiro MJ, Stoot JH, McCready VR, Bourguignon M, Mazière B. FDG accumulation and tumor biology. Nucl Med Biol. 1998;25:317-322.

17. Lovinfosse P, Weekers L, Bonvoisin C, Bovy C, Grosch S, Krzesinski JM, et al. Fluorodeoxyglucose F(18) positron emission tomography coupled with computed tomography in suspected acute renal allograft rejection. Am J Transplant. 2016;16:310-316.

18. Krejci K, Zadrazil J, Tichý T, Al-Jabry S, Horcicka V, Strebl $\mathrm{P}$, et al. Sonographic findings in borderline changes and subclinical acute renal allograft rejection. Eur J Radiol. 2009;71:288-295.

19. Yazdani M, Ghaemian N, Khafri S, Oliaei F. Can the kidney volume help to differentiate the types of rejection before biopsy? Caspian J Intern Med. 2019;10:11-15.

20. Park SB, Kim JK, Cho KS. Complications of renal transplantation: ultrasonographic evaluation. J Ultrasound Med. 2007;26:615-633.

21. Richard HM. Perirenal transplant fluid collections. Semin Intervent Radiol. 2004;21:235-237.

22. Horrow MM, Parsikia A, Zaki R, Ortiz J. Immediate postoperative sonography of renal transplants: vascular findings and outcomes. AJR Am J Roentgenol. 2013;201:W479-W486.

23. Zeisbrich M, Kihm LP, Drüschler F, Zeier M, Schwenger $\mathrm{V}$. When is contrast-enhanced sonography preferable over conventional ultrasound combined with Doppler imaging in renal transplantation? Clin Kidney J. 2015;8:606-614.

24. Meier M, Fricke L, Eikenbusch K, Smith E, Kramer J, Lehnert $\mathrm{H}$, et al. The serial duplex index improves differential diagnosis of acute renal transplant dysfunction. J Ultrasound Med. 2017;36:1607-1615.

25. Cano H, Castañeda DA, Patiño N, Pérez HC, Sánchez M, Lozano E, et al. Resistance index measured by Doppler ultrasound as a predictor of graft function after kidney transplantation. Transplant Proc. 2014;46:2972-2974.

26. Buturovic-Ponikvar J, Cerne S, Arnol M, Kandus A, Ponikvar R, Bren A. Early kidney graft size and Doppler parameters are associated with kidney graft function 1 year after transplantation. Transplant Proc. 2010;42:4026-4029.

27. Barba J, Rioja J, Robles JE, Rincón A, Rosell D, Zudaire $\mathrm{JJ}$, et al. Immediate renal Doppler ultrasonography findings $(<24 \mathrm{~h})$ and its association with graft survival. World J Urol. 2011;29:547-553.

28. Adibi A, Ramezani M, Mortazavi M, Taheri S. Color Doppler indexes in early phase after kidney transplantation and their association with kidney function on six month follow up. Adv Biomed Res. 2012;1: 62. doi: 10.4103/22779175.100184

29. Enhesari A, Mardpour S, Makki Z, Mardpour S. Early ultrasound assessment of renal transplantation as the valuable biomarker of long lasting graft survival: a crosssectional study. Iran J Radiol. 2014;11:e11492.

30. Elec FI, Lucan C, Ghervan L, Munteanu V, Moga S, Suciu M, et al. Ex-vivo perfusion machines in kidney transplantation. 
The significance of the resistivity index. Clujul Med 2014;87:27-29.

31. Seiler S, Colbus MS, Lucisano G, Rogacev KS, Gerhart MK, Ziegler $M$, et al. Ultrasound renal resistive index is not an organ-specific predictor of allograft outcome. Nephrol Dial Transplant. 2012;27:3315-3320.

32. Viazzi F, Leoncini G, Derchi LE, Pontremoli R. Ultrasound Doppler renal resistive index: a useful tool for the management of the hypertensive patient. J Hypertens. 2014;32:149-153.

33. Heine GH, Gerhart MK, Ulrich C, Köhler H, Girndt M. Renal Doppler resistance indices are associated with systemic atherosclerosis in kidney transplant recipients. Kidney Int. 2005;68:878-885.

34. Piscaglia F, Bolondi L; Italian Society for Ultrasound in Medicine and Biology (SIUMB) Study Group on Ultrasound Contrast Agents. The safety of Sonovue in abdominal applications: retrospective analysis of 23188 investigations. Ultrasound Med Biol. 2006;32:1369-1375.

35. Cokkinos DD, Antypa EG, Skilakaki M, Kriketou D, Tavernaraki E, Piperopoulos PN. Contrast enhanced ultrasound of the kidneys: what is it capable of? Biomed Res Int. 2013;2013:595873.

36. European Medicine Agency. 2017. Available from: URL: http://www.ema.europa.eu/docs/en_GB/document_library/ EPAR Summary for the public/human/000303/ WC500055374.pdf.

37. Tranquart F, Mercier L, Frinking P, Gaud E, Arditi M. Perfusion quantification in contrast-enhanced ultrasound (CEUS)--ready for research projects and routine clinical use. Ultraschall Med. 2012;33 Suppl 1:S31-S38.

38. Sidhu P, Cantisani V, Dietrich CF, Gilja OH, Saftoiu A, Bartels E, et al. The EFSUMB Guidelines and Recommendations for the Clinical Practice of Contrast-Enhanced Ultrasound (CEUS) in Non-Hepatic Applications: Update 2017 (Long Version). Ultraschall Med. 2018;39:e2-e44.

39. Farina R, Pennisi F, La Rosa M, Puglisi C, Di Benedetto A, Campisi G, et al. Functional study of the transplanted kidney with power Doppler US and time/intensity curves. Radiol Med. 2007;112:64-73.

40. Wang X, Yu Z, Guo R, Yin H, Hu X. Assessment of postoperative perfusion with contrast-enhanced ultrasonography in kidney transplantation. Int J Clin Exp Med. 2015;8:18399-18405.

41. Harvey CJ, Sidhu PS, Bachmann Nielsen M. Contrastenhanced ultrasound in renal transplant: application and future directions. Ultraschall Med. 2013;34:319-321.

42. Fischer T, Mühler M, Kröncke TJ, Lembcke A, Rudolph J, Diekmann F, et al. Early postoperative ultrasound of kidney transplants: evaluation of contrast medium dynamics using time-intensity curves. Rofo. 2004;176:472-477.

43. Grzelak P, Szymczyk K, Strzelczyk J, Kurnatowska I, Sapieha M, Nowicki M, et al. Perfusion of kidney graft pyramids and cortex in contrast-enhanced ultrasonography in the determination of the cause of delayed graft function. Ann Transplant. 2011;16:48-53.
44. Fischer T, Dieckhöfer J, Mühler M, Lembcke A, Morgera $\mathrm{S}$, Budde $\mathrm{K}$, et al. The use of contrast-enhanced US in renal transplant: first results and potential clinical benefit. Eur Radiol. 2005;15 Suppl 5:E109-E116.

45. Fischer T, Filimonow S, Dieckhöfer J, Slowinski T, Mühler M, Lembcke A, et al. Improved diagnosis of early kidney allograft dysfunction by ultrasound with echo enhancer--a new method for the diagnosis of renal perfusion. Nephrol Dial Treansplant. 2006;21:2921-2929.

46. Fischer T, Filimonow S, Rudolph J, Morgera S, Budde K, Slowinski T, et al. Arrival time parametric imaging: a new ultrasound technique for quantifying perfusion of kidney grafts. Ultraschall Med. 2008;29:418-423.

47. Bennozzi L, Cappelli G, Granito M, Davoli D, Favali D, Montecchi MG, et al. Contrast-enhanced sonography in early kidney graft dysfunction. Transplant Proc. 2009;41:12141215.

48. Jin Y, Yang C, Wu S, Zhou S, Ji Z, Zhu T, et al. A novel simple noninvasive index to predict renal transplant acute rejection by contrast-enhanced ultrasonography. Transplantation. 2015;99:636-641.

49. Fernandez PC, Ripolles T, Martinez. MJ, Blay J, Pallardó L, Gavela E. Diagnosis of acute cortical necrosis in renal transplantation by contrast-enhanced ultrasound: a preliminary experience. Ultraschall Med. 2013;34:340-344.

50. Kay HD, Mazonakis M, Geddes C, Baxter G. Ultrasonic microbubble contrast agents and the transplant kidney. Clin Radiol. 2009;64:1081-1087.

51. Schwenger V, Hankel V, Seckinger J, Macher-Göppinger $\mathrm{S}$, Morath $\mathrm{C}$, Zeisbrich $\mathrm{M}$, et al. Contrast-enhanced ultrasonography in the early period after kidney transplantation predicts long-term allograft function. Transplant Proc. 2014;46:3352-3357.

52. Mori G, GranitoM, Favali D, Cappelli G. Long-term prognostic impact of contrast-enhanced ultrasound and power Doppler in renal transplantation. Transplant Proc. 2015;47:2139-2141.

53. Stenberg B, Wilkinson M, Elliott S, Caplan N. The prevalence and significance of renal perfusion defects in early kidney transplants quantified using 3D contrast enhanced ultrasound (CEUS). Eur Radiol. 2017;27:4525-4531.

54. Mueller-Peltzer K, Negrão de Figueiredo G, Fischereder M, Habicht A, Rübenthaler J, Clevert DA. Vascular rejection in renal transplant: Diagnostic value of contrast-enhanced ultrasound (CEUS) compared to biopsy. Clin Hemorheol Microcirc. 2018;69:77-82.

55. Claudon M, Dietrich CF, Choi BI, Cosgrove DO, Kudo M, Nolsøe CP, et al. Guidelines and good clinical practice recommendations for contrast enhanced ultrasound (CEUS) in the liver - update 2012: a WFUMB-EFSUMB initiative in cooperation with representatives of AFSUMB, AIUM, ASUM, FLAUS and ICUS. Ultraschall Med. 2013;34:11-29.

56. Sis B, Sarioglu S, Celik A, Kasap B, Yildiz S, Kavukcu S, et al. Renal medullary changes in renal allograft recipients with raised serum creatinine. J Clin Pathol. 2006;59:377-381. 